\title{
Sodium cromoglycate in asthma: correlation between response and serum concentrations
}

\author{
Y YAHAV,${ }^{*}$ S DANY, $\dagger$ D KATZNELSON, ${ }^{*}$ AND Z FARFEL† \\ *Harry Shwachman Clinic, Department of Pediatrics ' $A$ ', + Clinical Pharmacology Unit and $\ddagger$ Department of \\ Medicine E, Chaim Sheba Medical Center, Tel-Hashomer, Sackler School of Medicine, Tel-Aviv University, \\ Israel
}

SUMMARY The clinical response to sodium cromoglycate treatment was compared with its concentration in serum. Twenty five children with asthma entered a 10 week trial of two baseline weeks followed by eight weeks of treatment by the inhalation of $20 \mathrm{mg}$ of sodium cromoglycate spincaps four times a day. Individual clinical response was determined by the differences between baseline and treatment periods of: $(a)$ percentage of symptomless days $(\Delta$ score 0$) ;(b)$ diary derived daily score for four symptoms ( $\Delta$ DS); and $(c)$ peak expiratory flow rate $(\Delta$ PEFR). At the end of the treatment period, patients inhaled a $20 \mathrm{mg}$ spincap of sodium cromoglycate and the technique of inhalation was graded. Concentrations of sodium cromoglycate in serum were measured by radioimmunoassay in samples withdrawn 5 to 120 minutes after inhalation. $\Delta$ Score $0, \Delta$ DS, and $\triangle$ PEFR correlated significantly with the area under the concentration time curve. Both the area under the sodium cromoglycate concentration time curve and clinical response correlated significantly with inhalation technique score. We suggest that response of children with asthma to inhalation treatment with sodium cromoglycate is correlated to its serum concentrations.

Sodium cromoglycate is an effective drug in the prophylactic treatment of asthma, especially in children. Its efficacy is ascribed to its ability to inhibit degranulation of mast cells in the respiratory tract. $^{1-4}$ It is claimed to be as effective as theophylline,${ }^{56}$ however, a quarter to a third of children appear not to respond to this treatment. ${ }^{78}$ This failure could presumably be due to either a true non-responsiveness to the drug or inadequacy in its delivery to target site.

Using radioimmunoassay to measure sodium cromoglycate in normal volunteers it has been shown that concentrations in the blood are very low after oral ingestion. ${ }^{9}$ Inhalation of the drug, however, led to much higher concentrations. ${ }^{9} 10$ We report the results of a prospective study in which the clinical response was compared with concentrations of sodium cromoglycate in serum that were obtained after inhalation of the drug.

\section{Patients and methods}

Twenty five children with chronic perennial asthma participated in the study. There were 21 boys and four girls whose ages ranged from 7 to 14 years, with a mean (SD) of $10 \cdot 4(1.9)$ years. Duration of asthma ranged from one to 12 years, with a mean of 6.3 years. Criteria for inclusion were a history of moderate to severe asthma that was poorly controlled while on routine treatment, needing repeated visits to the emergency room and recurrent hospital admission during the year preceding entry. Patients had a personal or family history of associated atopic disorders and reversibility of airway obstruction as shown by improvement of at least $15 \%$ in forced expiratory volume in one second $\left(\mathrm{FEV}_{1}\right) 15$ minutes after inhalation of $2.5 \mathrm{mg}$ salbutamol. None of the children had received sodium cromoglycate previously, nor had they been treated with corticosteroids in the three preceding months.

The study was conducted over a period of 10 weeks. The first two weeks were a baseline period during which the patients were instructed in the inhalation technique of capsules of sodium cromoglycate by a powder inhaler, the spinhaler. The patients were instructed to use the inhaler after a forced expiration, to keep their lips tightly attached to the mouthpiece of the device, to take a deep 
forceful inspiration to inhale the powder and to hold their breath for 10 seconds afterwards. Salbutamol inhalation was permitted as necessary. Theophylline was stopped in those using it in order to assess better the effect of sodium cromoglycate as a single prophylactic agent and to exclude improved compliance to theophylline as a cause for the results obtained. Over the next eight weeks, the children inhaled $20 \mathrm{mg}$ sodium cromoglycate spincaps four times a day while continuing to use salbutamol as needed.

The patients recorded the following symptoms in a daily diary on a $0-3$ scale of increasing severity: sleep disturbance, cough, wheeze, and impairment of activity. Score 3 was given for each of the following symptoms: severe sleepless night, persistent cough, severe wheezes, and physical disability. Score 0 was given when the patient was asymptomatic. Daily score was determined for each day by the sum of the individual scores given to each symptom (maximum score $=12$ ). Twice daily the patients also measured peak expiratory flow rates (PEFR) at home, using a Mini-Wright peak-flow meter. Patients were assessed clinically at entry to the study and at the end of weeks two, six, and 10. During each assessment patients were examined, PEFR was recorded as described above, and $\mathrm{FEV}_{1}$ was measured by a dry spirometer (Vitalograph). Patients were instructed to withhold the use of bronchodilators for at least 24 hours before their clinic visit. On the fourth visit, the patients inhaled one $20 \mathrm{mg}$ spincap of sodium cromoglycate. The technique of inhalation was assessed on a 0-3 scale taking into consideration strength and length of inspiration, motivation of performance, and positioning of patient's head and inhalation device. Venous blood samples for determination of sodium cromoglycate concentrations were drawn at $0,5,10,30,60$, and 120 minutes after inhalation.

Sodium cromoglycate concentration in the serum was determined by the radioimmunoassay method described by Brown et al. ${ }^{11}$ Plots of the concentrations versus time were constructed, and area under the curve was calculated by the trapezoidal method. ${ }^{12}$

Response to treatment was assessed by three parameters: score 0, daily score (DS), and PEFR. The percentage of symptomless days recorded in the diary was calculated for the baseline period and for the eight treatment weeks, and was termed score 0. Mean daily score was calculated from the patients' diaries for both baseline and treatment periods. Mean PEFR was calculated for baseline and treatment periods. For each patient $\Delta$ score 0 and $\Delta$ DS were calculated as the differences between individual score 0 , and mean daily score during baseline and treatment periods respectively. $\Delta$ PEFR was the difference between individual mean PEFR at treatment and baseline periods, divided by mean PEFR during baseline period and was expressed in per cent.

Results were expressed as mean (SD). Means were compared by Student's $t$ test, except for the comparison of means of daily score, where the Mann-Whitney test was used. ${ }^{13}$ A $p$ value of less than 0.05 was considered significant.

Informed consent was obtained from the parents. The local hospital ethics committee approved the study.

\section{Results}

Table I shows clinical and pharmacokinetic data of the patients. Treatment with sodium cromoglycate produced no side effects. Mean (SD) score 0 in the baseline period was $19.8(23.3) \%$ and in the treatment period $59.8(32.3) \%$ similar to the value obtained by Newth et al. ${ }^{14}$ The rise in mean score 0 during treatment is significant $(p<0 \cdot 002)$. There was no difference in mean score 0 after four or eight weeks of treatment. Mean daily score during the baseline and treatment periods, was $2.64(1 \cdot 1)$ and $0.85(1 \cdot 1)$ respectively $(p<0 \cdot 001)$. Cough and wheeze were the two symptoms which contributed most to the calculated daily score. Mean PEFR during the baseline period was $260(61) \mathrm{l} /$ minute and during treatment, 307 (76) $1 /$ minute $(p<0 \cdot 02)$. $\mathrm{FEV}_{1}$, which was measured only during the clinic visits, was $69 \cdot 3(15 \cdot 5) \%$ on admission to the trial, $78.6(19.5) \%$ at the end of the baseline period, and $89.5(18.2) \%$ at the end of the trial.

Serum sodium cromoglycate concentration determined after inhalation of $20 \mathrm{mg}$ of the drug rose rapidly. In 11 patients it reached a peak five minutes after inhalation, in eight patients 10 minutes after inhalation, and in five patients 15 minutes after inhalation. Peak concentrations varied considerably among the patients (table I). Mean peak sodium cromoglycate concentration was $51 \mu \mathrm{g} / \mathrm{l}$, and the mean value 120 minutes after inhalation was $13 \mu \mathrm{g} / \mathrm{l}$. The area under the sodium cromoglycate concentration time curve was calculated for each patient. Individual clinical response measured as the parameters $\Delta$ score $0, \Delta$ DS, and $\Delta$ PEFR were correlated with the pharmacokinetic parameters: area under the curve, peak sodium cromoglycate concentration, and the clinically convenient parameter of sodium cromoglycate concentration 10 minutes after inhalation (SCG 10 minute).

Table 2 shows that the correlation between $\Delta$ score 0 as well as $\triangle$ PEFR and all the pharmacokinetic parameters was significant, $\Delta$ DS corre- 
594 Yahav, Dany, Katznelson, and Farfel

Table 1 Clinical and pharmacokinetic data of patients

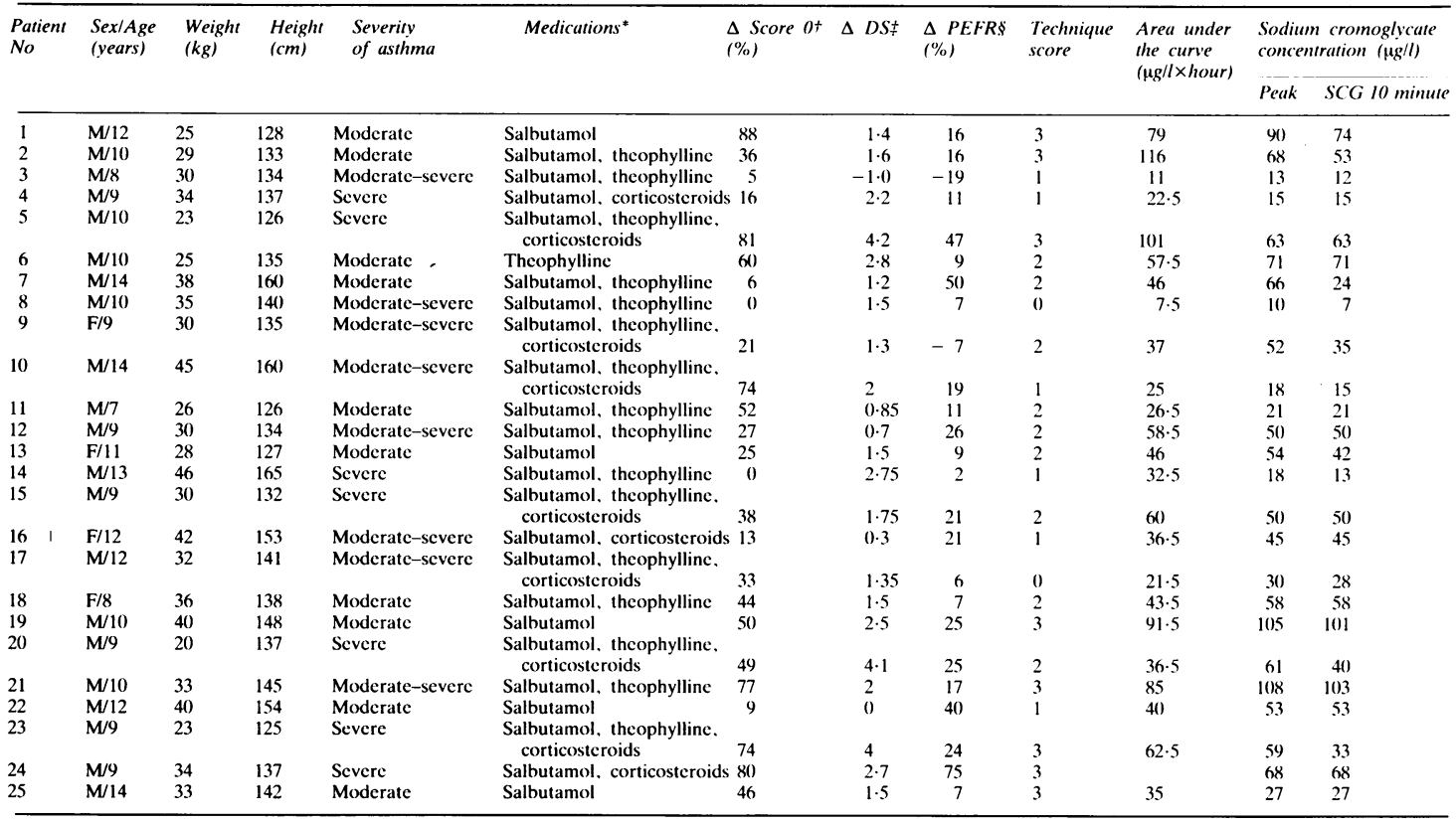

*Medications taken in the 12 months before sodium cromoglycate.

$\dagger \Delta$ Score $0=$ difference in percentage of symptomless days between baseline and treatment period; $\ddagger \Delta$ DS=difference in mean diary derived daily score for four symptoms between baseline and treatment period; $\$ \Delta$ PEFR=difference in mean peak expiratory flow rate between treatment and baseline period.

Table 2 Correlations between clinical and pharmacokinetic parameters

$r$ Value $p$ Value No of patients

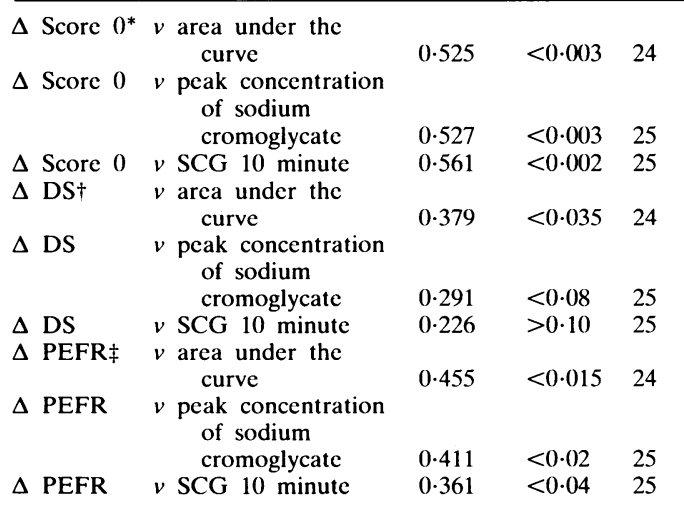

${ }^{*} \Delta$ Score $0=$ difference in percentage of symptomless days between baseline and treatment period; $+\Delta \mathrm{DS}=$ difference in mean diary derived daily score for four symptoms between baseline and treatment period; $\ddagger \Delta$ PEFR=difference in mean peak expiratory flow rate between treatment and baseline period. lated significantly only with the area under the curve. The correlation between $\Delta$ score 0 and SCG 10 minute is shown in fig 1 and the correlation between $\triangle$ PEFR and area under the curve is shown in fig 2 .

In order to determine whether the technique of inhalation of sodium cromoglycate affects clinical response to the drug, the pharmacokinetic parameters and clinical response were correlated with the efficiency of the technique of inhalation. Table 3 shows that highly significant correlations were obtained between the area under the curve, peak concentration of sodium cromoglycate, and SCG 10 minutes, and technique of inhalation. The correlation between the area under the curve and technique of inhalation is shown in fig 3 . There is also a correlation between clinical response and inhalation technique, but its significance is smaller (table 3).

Finally, in an attempt to assess the effect of improved delivery of sodium cromoglycate, four patients with low peak sodium cromoglycate concentration were reinstructed in the use of the inhaler. In three patients peak concentrations rose from $19(10) \mu \mathrm{g} / \mathrm{l}$ to $64(15) \mu \mathrm{g} / \mathrm{l}(\mathrm{p}<0 \cdot 025)$. In one patient it did not change. 


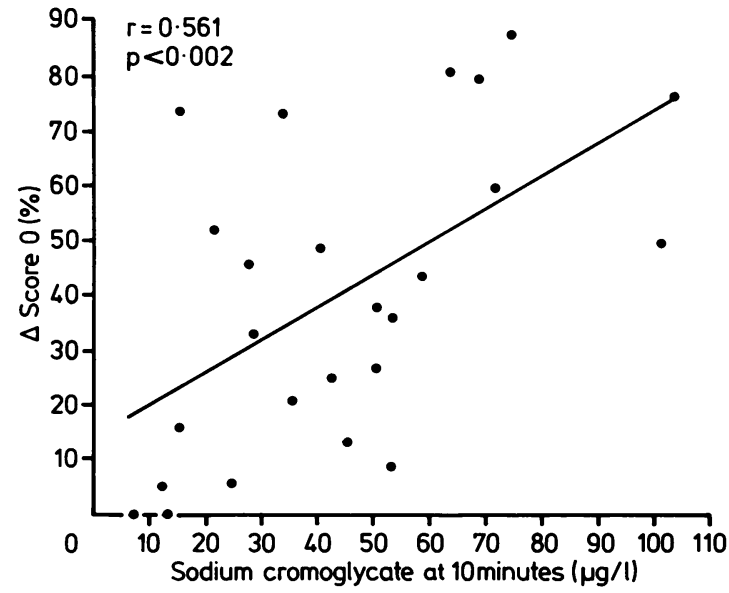

Fig 1 Correlation between the difference in percentage of symptomless days during baseline and treatment period $(\Delta$ score 0$)$ and concentration of sodium cromoglycate at 10 minutes.

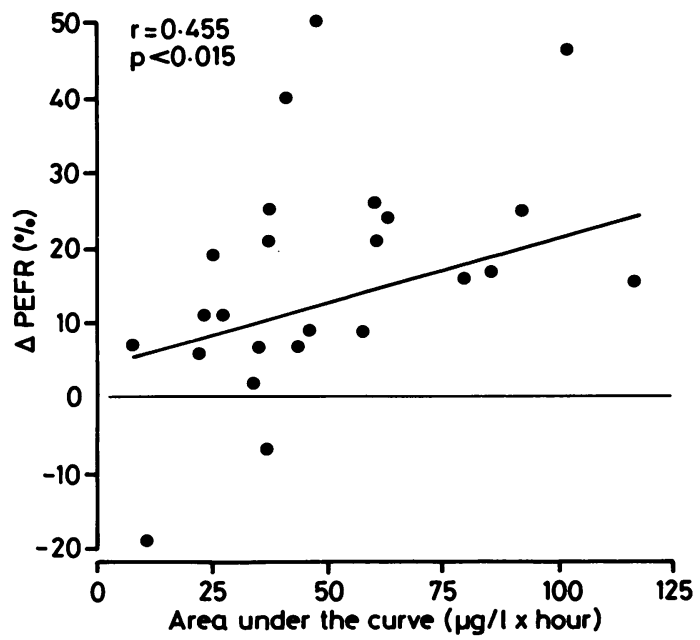

Fig 2 Correlation between the difference in mean peak expiratory flow rate at treatment and baseline periods $(\triangle P E F R)$ and sodium cromoglycate area under the concentration time curve.

\section{Discussion}

Measurement of sodium cromoglycate concentration enables one to determine the relation between drug concentration and response. As the site of action of this drug is not clinically accessible, measurement of sodium cromoglycate in the blood, apparently reflecting its concentration in the bronchioles, may be informative. Indeed, in normal
Table 3 Correlations between pharmacokinetic and clinical parameters and technique of inhalation

\begin{tabular}{|c|c|c|c|c|}
\hline & & $r$ Value & $p$ Value & $\begin{array}{l}\text { No of } \\
\text { patients }\end{array}$ \\
\hline \multicolumn{4}{|l|}{$\begin{array}{l}\text { Area under the } \\
\text { curve }\end{array}$} & 24 \\
\hline \multicolumn{5}{|c|}{$\begin{array}{l}\text { Peak concentration } \\
\text { of sodium }\end{array}$} \\
\hline cromoglycate & $v$ technique & $\begin{array}{r}0.717 \\
0.652\end{array}$ & $\begin{array}{l}<0.0005 \\
<0.0005\end{array}$ & 25 \\
\hline SCG 10 minute & $\begin{array}{l}v \text { technique } \\
v \text { technique }\end{array}$ & $\begin{array}{l}0.652 \\
0 \cdot 671\end{array}$ & $\begin{array}{l}<0.0005 \\
<0.0005\end{array}$ & $\begin{array}{l}25 \\
25\end{array}$ \\
\hline$\Delta \mathrm{DS} \dagger$ & $v$ technique & 0.410 & $<0.02$ & 25 \\
\hline$\Delta$ PEFR $\ddagger$ & $v$ technique & 0.373 & $<0 \cdot 035$ & 25 \\
\hline
\end{tabular}

* $\Delta$ Score $0=$ difference in percentage of symptomless days between baseline and treatment period; $† \Delta \mathrm{DS}=$ difference in mean diary derived daily score for four symptoms between baseline and treatment period; $\ddagger \Delta$ PEFR=difference in mean peak expiratory flow rate between treatment and baseline period.

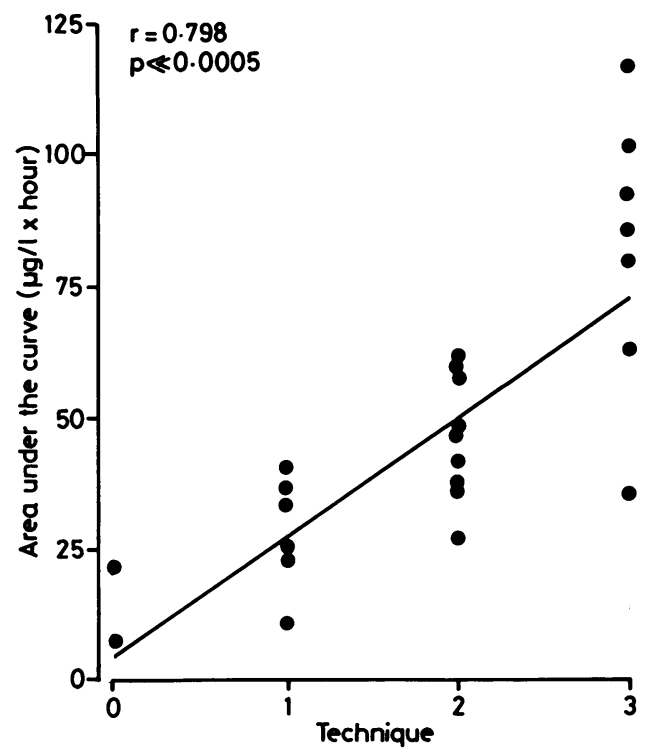

Fig 3 Correlation between sodium cromoglycate area under the concentration time curve and technique of inhalation.

volunteers it was shown that the concentrations in the blood rise rapidly after inhalation, ${ }^{9}{ }^{10}$ whereas after oral ingestion blood concentrations are low, ${ }^{9}$ thereby explaining the ineffectiveness of oral sodium cromoglycate in asthma.

Serum sodium cromoglycate concentrations in our young asthmatic patients rose after inhalation of $20 \mathrm{mg}$ sodium cromoglycate and reached a mean peak of $51 \mu \mathrm{g} / \mathrm{l}$, compared with a mean 
peak of $46 \mu \mathrm{g} / \mathrm{l}$ observed in adult volunteers. ${ }^{9}$ Concentrations declined promptly to a mean of $13 \mu \mathrm{g} / \mathrm{l}$ at 120 minutes after inhalation, in a similar way to that observed in volunteers. ${ }^{9-11}$ In contrast, Patel et al found that in asthmatic adults four successive inhalations of $5 \mathrm{mg}$ sodium cromoglycate produced peak concentrations of only $5 \mu \mathrm{g} / \mathrm{l}$ which did not decline for 60 minutes after inhalation. ${ }^{15}$ The reasons for this discrepancy are not clear.

A correlation was found between individuals' response and pharmacokinetic parameters. The $\Delta$ score 0 correlated significantly with all three pharmacokinetic parameters. The correlation between $\Delta$ PEFR and these three parameters was also significant, although of lower magnitude. The $\Delta$ DS correlated significantly only with the area under the curve which integrates concentration over time.

Spontaneous improvement during baseline period was observed as shown by the $\mathrm{FEV}_{1}$ values, however, the correlation which was found between the response during the treatment period and the pharmacokinetic parameters, argues for a direct relation between serum sodium cromoglycate concentration and the clinical response observed. Furthermore, this correlation provides evidence for the efficacy of sodium cromoglycate in this group of asthmatic children.

In an attempt to define the basis for the low sodium cromoglycate concentration in some patients, the technique of sodium cromoglycate inhalation was correlated with blood concentrations and with clinical response. Both pharmacokinetic parameters and clinical response correlated significantly with technique of inhalation. Brown et al have found that in normal volunteers the sodium cromoglycate area under the curve correlated with peak inspiratory flow rate, however, they state that variation in area under the curve and peak sodium cromoglycate is not solely explained by variation in peak inspiratory flow rate. ${ }^{9}$ Similar data of intrasubject and intersubject variability were recently reported by Neale et al. ${ }^{16}$ If technique of inhalation in someway or partly reflects peak inspiratory flow rate (which was not measured in our patients), one could argue that low sodium cromoglycate serum concentrations in some patients were the result rather than the cause of the poorer respiratory performance in the poorly responding patients. Peak inspiratory flow rate, however, falls only in severe obstructive airway disease, which did not occur in our patients. In addition, clinical response to sodium cromoglycate in our study was not related to the initial severity of disease. Two of seven patients with severe disease were poor responders ( $\Delta$ score $0<20 \%$ ); this is similar to the proportion of poor responders in all the patients (seven out of $25,28 \%)$.

It is not inconceivable, however, that severe airway obstruction may impede delivery of sodium cromoglycate to its target site of action. In such circumstances the use of a bronchodilator before inhalation of this drug or the use of special inhalation devices ${ }^{17}$ may be considered and studied. No side effects of sodium cromoglycate were observed in this study, neither were dose related side effects observed in worldwide experience over many years. ${ }^{3}$ Because response seems to be related to sodium cromoglycate concentrations in the blood, administration of larger doses than the recommended ones should also be considered.

This study shows that response to sodium cromoglycate is correlated to its concentration in the blood. Most of the non-responding patients have decreased concentrations of the drug, and in some of them this can be improved by additional training in proper use of the inhaler. There may yet be a few patients who do not respond even to apparently therapeutic concentrations of sodium cromoglycate.

This work was supported by Trupharm Ltd.

\section{References}

1 Altounyan RE. Review of clincal activity and mode of action of sodium cromoglycate. Clin Allergy 1980;10:481-9.

2 Berman BA. Cromolyn: past, present and future. Pediatr Clin North Am 1983;30:915-30.

${ }^{3}$ Shapiro GG, Konig P. Cromolyn sodium: a review. Pharmacotherapy 1985:5:156-170.

4 Bernstein IL. Cromolyn sodium in the treatment of asthma: coming of age in the United States. J Allergy Clin Immunol 1985;76:381-8.

5 Edmunds AT, Carswell F, Robinson P, et al. Controlled trial of cromoglycate and slow-relcase aminophylline in perennial childhood asthma. Br Med J 1980;281:842.

${ }^{6}$ Furukawa CT, Shapiro GG, Bicrman CW, Kraemer MJ, Ward DJ, Pierson WE. A double-blind study comparing the effectiveness of cromolyn sodium and sustained-release theophylline in childhood asthma. Pediatrics 1984;74:453-9.

7 Silverman M, Connolly NM, Balfour-Lynn L, Godfrey S. Longterm trial of disodium cromoglycate and isoprenaline in children with asthma. $\mathrm{Br}$ Med J 1972;iii:378-81.

* Godfrey S, Balfour-Lynn L, Konig P. The place of cromolyn sodium in the long-term management of childhood asthma based on a 3 to 5 year follow up. J Pediatr 1975;87:465-73.

9 Brown K, Hodder RW, Neale MG. Plasma concentrations of sodium cromoglycate in volunteers determined by a new method. Br J Clin Pharmacol 1981;11:425.

16) Fuller RW, Collier JG. The pharmacokinetic assessment of sodium cromoglycate. J Pharm Pharmacol 1983;35:289-92.

11 Brown K, Gardner JJ, Lockley WJS, Preston JR, Wilkinson DJ. Radioimmunoassay of sodium cromoglycate in human plasma. Ann Clin Biochem 1983;20:31-6.

12 Gibaldi M, Perrier D. Pharmacokinetics. New York: Marcel Dekker, 1975.

13 Zarr JH. Biostatistical analysis. Englewood Cliffs, NJ: PrenticeHall, 1974.

14 Newth CJL, Newth CV, Turner JAP. Comparison of nebulized sodium cromoglycate and oral theophylline in controlling 
symptoms of chronic asthma in pre-school children: a doubleblind study. Aust NZ J Med 1982;12:232-8.

15 Patel KR. Tullet WM, Neale MG, Wall RT. Tan KM. Plasma concentrations of sodium cromoglycate given by nebulisation and metered dose inhalers in patients with exercise-induced asthma: relationship to protective effect. Br J Clin Pharmacol 1986;21:231-3

16 Neale MG. Brown K. Hodder RW, Auty RM. The pharmacokinetics of sodium cromoglycate in man after intravenous and inhalation administration. Br J Clin Pharmacol 1986;22:373-82.
${ }^{17}$ Levison H, Reilly PA. Worsely GH. Spacing devices and metered-dose inhalers in childhood asthma. $J$ Pediatr 1985:107:662-8.

Correspondence and request for reprints to Dr Z Farfel, Clinical Pharmacology Unit. The Chaim Medical Center, Tel-Hashomer 52621, Israel

Accepted 30 November 1987 